\title{
An ab-initio study of multiple conformers of glycine
}

\author{
Dan Kaplan ${ }^{1}$, Greg Recine, and H. Zhang \\ Stevens Institute of Technology, \\ Hoboken, NJ 07030 USA \\ ${ }^{1}$ email: dkaplan@stevens .edu
}

\begin{abstract}
The recent combination of new computational chemistry techniques and high performance computational hardware is allowing unprecedented levels of accuracy in the calculations of physical quantities such as potential energy surfaces and rotational-vibrational spectra. Previous results exist in the literature for the first three most stable conformers of glycine at the aug-cc-pVDZ basis set. In this work, we extend the known results, presenting calculations of the four most stable conformers of glycine using the aug-cc-pVQZ basis set. We compare our calculations to experimental values and show that our current calculations differ by $<2 \%$ from measured values, much better than results from previous years. When searching for molecules in the Interstellar Medium this small difference suggests that computational methods are becoming well-suited for the task. The natural question to ask is: at what point will the small deviation from experimental values render our computations just as reliable as experiments? We feel that the current results show that we are indeed close to this goal.
\end{abstract}

Keywords. Methods: numerical, molecular data, astrochemistry

\section{Introduction}

Glycine $\left(\mathrm{HO}_{2} \mathrm{CCH}_{2} \mathrm{NH}_{2}\right)$, one of the twenty amino acids and the building blocks of proteins, is a biological compound which has great importance in many fields. Specifically, we are interested in the debate over the possible presence of glycine in the interstellar medium (Kuan et al. 2003, Snyder et al. 2005). If glycine were indeed present it would have obvious and far-reaching implications to Astrobiology. It is important, therefore, that we be able to definitively resolve this debate. This work aims to alleviate some confusion on this topic by providing more accurate calculations of the vibrational spectrum of glycine. With this improved knowledge any identification of glycine will be more definitive. In addition, we have provided results for the less-studied fourth lowest energy conformer of glycine, for this may be present in the interstellar medium as well.

\section{Methods and Results}

We have used the computational chemistry software package GAMESS (Schmidt et al. 1993, Gordon \& Schmidt 2005) to calculate the vibrational spectrum of glycine. These calculations have been performed at two separate levels of computational sophistication: CCSD(T) with a 6-31G basis set and DFT-B3LYP with an aug-cc-pVDZ basis set (Woon \& Dunning 1993). The results are summarized below and compared with previous results (Stephanian et al. 1998). 
Table 1. Percent differences between calculated and observed values

\begin{tabular}{c|c|c|c|c}
\hline Computation $\backslash$ Conformer & I & II & III & IV \\
\hline \hline aug-cc-pVDZ & $1.96 \%$ & $3.86 \%$ & $2.26 \%$ & $2.51 \%$ \\
\hline $\mathrm{CCSD}(\mathrm{T})$ & $1.95 \%$ & $2.56 \%$ & $2.38 \%$ & $2.07 \%$ \\
\hline Stephanian et. al. & $\mid 4.17 \%$ & $4.45 \%$ & $4.6 \%$ & $\mathrm{~N} / \mathrm{A}$ \\
\hline
\end{tabular}

\section{Conclusions}

We have provided the highest level of accuracy calculations for the four most stable conformers of glycine. We predict these calculations will help aid the debate about the presence of glycine in the interstellar medium by providing researchers with the best information available concerning the spectrum of glycine. We will also entertain the notion that computational methods for calculating vibrational spectra are becoming so reliable as to render experiments in this area obsolete.

\section{References}

Kuan, Y.-J., Charnley, S. B., Huang, H.-C., Tseng, W.-L., \& Kisiel, Z. 2003, ApJ, 593, 848

Gordon, M. S. \& Schmidt, M. W. 2005, in: C.E.Dykstra, G. Frenking, K. S. Kim, \& G.E.Scuseria (eds.), Theory and Applications of Computational Chemistry, the first forty years, (Amsterdam: Elsevier)

Schmidt, M. W., et al. 1993, J. Comput. Chem., 14, 1347

Snyder, L., Lovas, F., Hollis, J., Friedel, D., Jewell, P., Remijan, A., Ilyushin, V., Alekseev, E., \& Dyubko, S. 2005, ApJ, 619, 914

Stephanian, S., Reva, I., Radchenko, E., Rosado, M., Duarte, M., Fausto, R., \& Adamowicz, L. 1998, J. Phys. Chem. A, 102, 1041

Woon, D. E. \& Dunning, T. H. 1993, J. Chem. Phys., 98, 1358 